\title{
Challenges for Anticoagulation in Atrial Fibrillation
}

Ana Inês da Costa Bronchtein

Hospital Copa D'or - Rio de Janeiro, RJ - Brazil

Atrial fibrillation (AF) is the most prevalent arrhythmia in the world. ${ }^{1}$ It is considered a current epidemic and one of the main causes of ischemic stroke, usually with severe and debilitating conditions, and is responsible for systemic cardioembolic phenomena, increased number of cases of congestive heart failure (CHF), cognitive impairment related to silent embolic phenomena, and increased overall mortality in affected patients.

Vitamin $\mathrm{K}$ antagonists (VKA) represented in the vast majority of studies with warfarin have been widely used for many years; however, they have complex adherence due to several factors, including the need for frequent INR monitoring, regular dose adjustments for maintaining a suitable time in therapeutic range (TTR) $(>=70 \%)$ and interactions with a number of drugs and foods, which contributed to its ever decreasing use. ${ }^{2}$ Direct-acting anticoagulants (DACs), represented by dabigatran, rivaroxaban, apixaban and, more recently, edoxaban, offer an alternative to VKA, without the disadvantages presented by the latter.

DACs have been increasingly used for the prevention of ischemic stroke and systemic embolic phenomena in non-valvular AF, with efficacy and safety confirmed in randomized multicenter non-inferiority studies with an overwhelming number of patients included. ${ }^{3}$ Besides, in real-life studies, the results of phase III studies have been confirmed, demonstrating advantages of using DACs even in older populations, with confirmed reduction in intracranial hemorrhage and some DACs demonstrating superiority in mortality compared to warfarin.

Nevertheless, although the introduction of DACs has promoted the use of anticoagulation in patients

\section{Keywords}

Atrial Fibrillation; Anticoagulants; Brain Ischemia; Vitamin K; Thromboembolism/ prevention and control. with non-valvular AF (absence of moderate to severe mitral stenosis and/or presence of mechanical valve), and despite the availability of national and international guidelines on the subject, ${ }^{4-6}$ the application of these guidelines in clinical practice is still far from desired worldwide, either due to the use of incorrect doses (usually below the recommended ones) or to inadequate use due to lack of knowledge of related drug interactions and fear of bleeding in the older population.

In this retrospective observational study, which collected data from an electronic medical record, conducted at a single center of a private tertiary hospital in Salvador (BA), Geraldes et al. ${ }^{7}$ evaluated the predictors of oral anticoagulation in patients with non-valvular AF and atrial flutter from 2011 to 2016 and how DACs are being incorporated in this context. A well-written original article where the authors evaluated 377 patients, mostly with paroxysmal $\mathrm{AF}$, with a high rate of comorbidities such as: SAH, DM, history of HF, AMI and stroke were separated into two groups: with and without anticoagulants. The anticoagulated group was divided into use of DACs and warfarin, and the following variables were listed: previous paroxysmal $\mathrm{AF}$, presence of $\mathrm{CHF}$, serum creatinine, EF, LA diameter, presence of biological prosthesis, moderate valvular disease and history of previous electrical or chemical cardioversion.

The authors demonstrated that, during these 5 years of follow-up, there was a significant increase in the number of patients anticoagulated with DACs (from $29 \%$ to $70 \%$ - a relative increase of $144.8 \%$ and an annual incorporation of $10.4 \%$ ) and a progressive decrease in warfarin use (36\% to $17 \%)$ in the population evaluated, with a drop in antiplatelet use alone (21\% to $6 \%$ ), confirming the initial impression that the increase in DAC prescriptions has accelerated the decline in warfarin prescriptions. It is worth noting that the use of antiplatelets alone in AF does not protect from ischemic stroke/thromboembolism (ET) in

Mailing Address: Ana Inês da Costa Bronchtein

Rua Rainha Guilhermina, 23/401. Postal Code: 22441120, Leblon Rio de Janeiro - RJ - Brazil.

E-mail: a.bronchtein@gmail.com

DOI: https://doi.org/10.36660/ijcs.20190003 
patients with high CHA2DS2VASc and high HAS-BLED (prevalent study population), and increases the risk of bleeding in this population.

In the study by Geraldes et al., 7 the variables previous AF episode $(\mathrm{p}<0.001)$, hypertension $(\mathrm{p}<0.001)$ and low HAS-BLED score were predictors of anticoagulation, while increased serum creatinine $(p<0.002)$, increased LA $(p=0.003)$ and presence of biological prosthesis $(p=0.007)$ were predictors inversely associated with the prescription of DACs, i.e., they were predictors of warfarin prescription. Each $1 \mathrm{mg} / \mathrm{ml}$ increase in serum creatinine led to $82 \%$ less chance of patients using DACs, reflecting the rejection of anticoagulating patients with impaired renal function despite the safety demonstrated in patients with clearance of up to $30 \mathrm{mg} / \mathrm{ml}$ with all DACs. They also demonstrated a high correlation between HAS-BLED and CHA2DS2VASc in the study population, which reflects the reality of AF patients with a high rate of associated comorbidities and a high risk of both thromboembolic events and major bleeding.

Of the total population evaluated in the study, 75\% were discharged on oral anticoagulation (20\% VKA and $55 \%$ DACs) and the vast majority had a history of AF $(p=0.001)$ and TIA/stroke $(p=0.008)$, were elderly $(\mathrm{p}=0.005)$ and had smaller HAS-BLEDs with higher weights. Surprisingly, 93 patients referred for anticoagulation were discharged without using such drugs and, in this group, $54 \%$ (50 patients) had no reason for not using them or the reasons were inconsistent with the medical literature.

The authors also point out, which seems extremely relevant, that some physicians did not apply the CHA2DS2VASc risk score to assess patients' thromboembolic risk. Instead, they used only their clinical impression, which is often inaccurate, although the population's average CHA2DS2VASc was as high as 3.4 +-2. Of the 208 patients on DACs, $63(30 \%)$ had inadequate prescriptions for severe interactions and 58 patients were prescribed inadequate doses for the patient profile. Doses below effective levels were the most common error found.

Marzec et al., ${ }^{8}$ in an article published in the JACC in 2017 (7), studied 655,000 patients with risk score CHA2DS2VASc $>1$ in the PINNACLE registry, where they analyzed the use of warfarin and DACs in non-valvular AF. The authors also concluded that the introduction of DACs in clinical practice was associated with improved rates of anticoagulation for AF but many gaps were still to be filled and variations in clinical practice were quite inconsistent regarding anticoagulation with DACs. These authors reported that DACs were preferably used in patients with few comorbidities, low risk of ischemic stroke and in those previously anticoagulated with warfarin. The authors suggested that further studies would be needed to better define the factors associated with variations and underuse of DACs in patients with high risk of ischemic stroke, emphasizing the importance of applying specific strategies to reduce the risk of ischemic stroke in patients with AF.

Monelli et al., ${ }^{9}$ in a single-center prospective observational real-life Italian study assessed records of patients using DACs (the REGINA study - registry of patients on non-vitamin $\mathrm{K}$ oral anticoagulants), which included 227 patients with mean age of 81.6 years (about $80 \%>80$ years of age) and mean CHA2DS2vasc of 5 and HAS-BLED of 4, with mean clearance of 59.2 and concluded that in a population of elderly and clinically complex patients, especially octogenarians, a population that is similar to the study discussed here, DACs were safe and effective and the careful follow-up of these elderly people with a high treatment adherence rate contributed to better prognosis in this population (8).

Another study evaluating anticoagulation in $\mathrm{AF}$, the ORBIT-AF study (outcomes registry for better quality of care in the treatment of AF) showed a higher prevalence of DAC use among AF patients seen by electrophysiologists compared with clinical cardiologists and primary care physicians or generalists. This is probably due to the fact that electrophysiologists receive the referral of a larger number of patients without contraindication for anticoagulation, because after an ablative procedure, full anticoagulation is recommended, i.e., they deal with a previously selected population. ${ }^{10}$

In conclusion, the study by Geraldes et al., ${ }^{7}$ realistically portrays the current situation of AF anticoagulation in our community, highlighting an important advance in the use of anticoagulation, especially DACs, but also drawing attention to improvements in this practice, which requires significant and urgent changes in order to use oral anticoagulation at discharge in this population with higher thromboembolic risk as a quality factor in $\mathrm{AF}$ care and an important indicator of primary prevention in public health. 


\section{References}

1. Fuster V, Ryden LE, Cannom DS, Crijns HJ, Curtis A, Kenneth A, et al. ACC/AHA/ESC 2006 guidelines for the management of patients with atrial fibrillation -- executive summary: a report of the American College of Cardiology/American Heart Association Task Force on Practice Guidelines and the European Society of Cardiology Committee for Practice Guidelines (Writing Committee to Revise the 2001 Guidelines for the Management of Patients with Arial Fibrillation). J Am Coll Cardiol 2007;50(6):562

2. Birman-Deych E, Radford MJ, Nilasena DS, Gage BF. Use and effectiveness of warfarin in Medicare beneficiaries with atrial fibrillation. Stroke 2006;37(4):1070-4.

3. Heidbuchel H, Verhamme P, Alings M, Camm AJ. Updated European Heart Rhythm Association practical guide on the use of non-vitamin-K antagonist anticoagulants in patients with non-valvular atrial fibrillation: Executive summary. ESC Scientific Document Group. Eur Heart J. 2017 Jul 14; 38(27): 2137-49.

4. Magalhães LP, Figueiredo MJ, Cintra FD. Executive Summary of the II Brazilan Guidelines for Atrial Fibrillation.II Brazilian Guidelines for Atrial Fibrillation. Arq Bras Cardiol.2016;107(6):501-8.

5. Kirchhof P, Benussi S, Kotecha D, (Task force members). 2016 Guidelines for the management of atrial fibrillation developed in collaboration with EACTS. Eur Heart J. 2016; 37(38): 2893-962.
6. January CT, Wann S, Calkins H, Chen LY, Cigarroa JE, Cleveland Jr JC, et al. 2019 Focused Update on Atrial Fibrillation. 2019 AHA/ACC/ HRS Focused Update of the 2014 AHA/ACC/HRS Guideline for the Management of Patients With Atrial Fibrillation. Circulation. 2019; 140(2):e125-e151.

7. Geraldes MFA, Darze ES, Rocha PN. Trends and predictors of oral anticoagulation in patients with atrial fibrillation: a serial cross-sectional study from 2011 to 2016. Int J Cardiovasc Sci. 2020;33(1):68-78.

8. Marzec LN, Wang J, Shah, Chan PS, Ting HH, Gosch KL, et al. Influence of direct oral anticoagulants on rates of oral anticoagulation for atrial fibrillation. J Am Coll Cardiol. 2017;69(20):2475-83.

9. Monelli M, Molteni M, Cassetti G, Bagnara L, Del Grazia V, Zingale L, et al. Non- Vitamin K oral anticoagulante use in the elderly: a prospective real-world study - data from the REGIstry of patients on Non-vitamin K oral anticoagulants (REGINA). Vasc Health Manag. 2019;15:19-25.

10. Fosbol L, Holmes DN, Piccin JP, Hplmes DN, Piccini JP, Thomas L, Reiffe JA, Mills RN, et al. Provider specialty and atrial fibrillation treatment strategies in United States Community practice: findings from the ORBITAF registry. J Am Heart Assoc. 2013;2(4):e000110. 